\title{
Environments of nearby quasars in Sloan Digital Sky Survey
}

\author{
H. Lietzen ${ }^{1}$, P. Heinämäki ${ }^{1}$, P. Nurmi ${ }^{1}$, E. Tago ${ }^{2}$, E. Saar ${ }^{2}$, J. Liivamägi ${ }^{2}$, E. Tempel ${ }^{2}$, M. Einasto $^{2}$, J. Einasto $^{2}$, \\ M. Gramann ${ }^{2}$, and L. O. Takalo ${ }^{1}$ \\ 1 Tuorla Observatory, Department of Physics and Astronomy, University of Turku, Väisäläntie 20, 21500 Piikkiö, Finland \\ e-mail: heilie@utu.fi \\ 2 Tartu Observatory, 61602 Tõravere, Tartumaa, Estonia
}

Received 8 January 2009 / Accepted 24 March 2009

\section{ABSTRACT}

\begin{abstract}
Context. For the first time, spectroscopic galaxy redshift surveys are enabling galaxies to be studied with the nearest quasars. This allows the dependence of the activity of a quasar on its environment to be studied in a more extensive way than before.

Aims. We study the spatial distribution of galaxies and properties of groups of galaxies in the environments of low redshift quasars in the Sloan Digital Sky Survey (SDSS). Our aim is to understand how the nearby quasars are embedded in the local and global density field of galaxies and how the environment affects quasar activity.

Methods. We analyze the environments of nearby quasars using number counts of galaxies. We also study the dependence of group properties on their distance to the nearest quasar. The large-scale environments are studied by analyzing the locations of quasars in the luminosity density field.

Results. Our study of the number counts of galaxies in quasar environments shows an underdensity of bright galaxies at a few Mpc from quasars. Groups of galaxies with a quasar closer than $2 \mathrm{Mpc}$ are also poorer and less luminous than average. Our analysis of the luminosity density field shows that quasars clearly avoid rich superclusters. Nearby quasars seem to be located in the outskirts of superclusters or in the filaments connecting them.

Conclusions. Our results suggest that quasar evolution may be affected by density variations both on supercluster scales and in the local environment.
\end{abstract}

Key words. quasars: general - galaxies: general

\section{Introduction}

Active galactic nuclei (AGN) are believed to have evolved by mergers between galaxies that cause an inflow of gas toward the galaxy center that in turn fuels the growth of the central black hole. According to the theory of quasar formation by Hopkins et al. (2005b), mergers frequently occur between galaxies during the hierarchial growth of structure. Mergers that involve gas-rich galaxy progenitors produce inflows of gas that cause starbursts. High gas densities fuel the black-hole growth, and the galaxy is seen as a luminous infrared galaxy (LIRG). As the black-hole mass increases, the feedback energy starts to expel the gas fueling accretion. The galaxy is then seen as an optical quasar. Finally, the feedback of the active galactic nucleus terminates further black-hole growth, after which the galaxy becomes an ordinary galaxy containing a dead quasar. Although this is the prevailing paradigm for quasar evolution, it is also possible that it may not be the only mechanism in initiating activity for all types of active galaxies. Activity may also be triggered by secular processes, such as bar instabilities (Sellwood \& Moore 1999).

One way of testing different theories for nuclear activity is to study the environments of AGN. If mergers are important to quasar activity, then the environments of quasars should allow mergers to happen. In clusters of high velocity dispersion, galaxies have insufficient time to merge. Clusters are less likely to host quasars than small groups of galaxies, which usually have lower velocity dispersions (Hopkins et al. 2005b).

It is well known that environmental density affects the properties of galaxies, as found for example by the morphology-density relation (Dressler 1980). This dependency was studied, e.g., by Kauffmann et al. (2004). Their results indicated that the stellar mass of galaxies increases and their star formation rate decreases with the growth of the local density. They also found that a higher fraction of galaxies hosts AGN in low-density environments. The morphologies, colors, and starformation rates of galaxies are clearly related to the assembly history of their halos (Maulbetsch et al. 2007).

Another reason for studying the environments of quasars is to test the unification schemes of different types of objects (Coldwell \& Lambas 2006). Different types of AGN are thought to be physically similar objects that are viewed from different directions (Antonucci 1993). If this is true, all AGN should have similar environments since the properties of their environments do not depend on the viewing angle. It is also possible that mergers are more important to the evolution in some types of AGN, while others might be triggered by other mechanisms. The activity and luminosity of an AGN may play an important role that provide clues about their merger history and associated environment.

Earlier studies of AGN environments used mostly galaxy counts in the fields of individual objects. The first results were from Bahcall et al. (1969), who showed that five quasars lie within the approximate geometrical boundaries of clusters of galaxies. In later work, the spatial galaxy-galaxy crosscorrelation amplitude was estimated in ways similar to those initiated by Longair \& Seldner (1979). The correlation studies have shown that quasars mostly occupy enhanced local densities, such as groups or clusters of galaxies. For example, 
De Robertis et al. (1998) found that the environments of Seyfert 2 type galaxies not differ from the control sample of normal galaxies, while the environments of Seyfert 1 galaxies are clearly poorer. The environments of BL Lac objects were studied by Smith et al. (1995) and Wurtz et al. (1997). Both these studies found that BL Lac objects lie in poor environments, usually in clusters of Abell richness class 0 or less. According to McLure \& Dunlop (2001), radio galaxies are distributed quite evenly over all cluster richness levels, but quasars are more frequent in poor environments. This was verified by Wold et al. (2000), who demonstrated that although there are a few quasars in rich environments, most of them seem to prefer galaxy groups or clusters of Abell richness class 0. To summarize, most AGN lie in very poor clusters, and have only a few more companion galaxies than found in the background field, on a few hundred kpc scales.

The Sloan Digital Sky Survey (SDSS) offers new possibilities for studying the large-scale structure of the Universe. For studying the environments of quasars, SDSS contains not only data for detailed studies of individual targets, but the data that it provides for a large number of quasars and galaxies makes it possible to study these environments on larger scales than before.

Li et al. (2006) measured AGN-galaxy cross-correlations for a large sample of quasars and galaxies in the SDSS Data Release 4. They found that on scales of between $100 \mathrm{kpc}$ and $1 \mathrm{Mpc}$ AGN hosts are clustered more weakly than inactive galaxies, while on smaller scales their clustering is slightly stronger. Their explanation of this result is that AGN reside preferentially at the centers of dark matter halos.

A slightly different approach was used by Miller et al. (2003), who studied the fraction of galaxies that possess an AGN, as a function of the environment. They considered galaxies in the redshift range $0.05 \geq z \geq 0.095$ of absolute magnitude $M<-20.0$ from the Early Data Release of the SDSS. Their main result was that $\sim 40 \%$ of galaxies in their sample have an AGN, and they did not find any relation between the AGN fraction and local galaxy density.

One of the most extensive studies of the environments of nearby quasars was completed by Coldwell \& Lambas (2006). They used quasars and galaxies at redshifts lower than 0.2 in the third Data Release of the SDSS to study the properties of galaxies in the environments of quasars. Their main results were that nearby quasars avoid high density regions, and that galaxies close to quasars usually have a disc-type morphology and a high star-formation rate.

As the data volume provided by the SDSS has increased, the environments of quasars have become an increasingly popular subject to study. One of the latest studies was completed by Strand et al. (2008), who compared the environments of quasars to those of AGN selected from the photometric galaxy sample of the SDSS Data Release. The galaxy densities were measured using data from the photometric catalog, which contains far more galaxies than the spectroscopic sample, but the lower redshift accuracy limits their analysis. Their main result is that quasars lie in higher density regions than AGN on scales of smaller than 2 Mpc.

We extend these previous studies to galaxy group and supercluster scales, and study the environments of quasars on three different scales. First, we concentrate on galaxies around quasars by analyzing the number density of galaxies at different distances from quasars. On the next scale, we study the relations between the group properties and the distance of the closest quasar. On the largest scale in this study, we use the luminosity-density fields, constructed from the galaxy catalogs. This allows us to describe the locations of quasars on supercluster scales. Throughout this work, we assume that $\Omega_{\mathrm{m}}=0.3$, $\Omega_{\Lambda}=0.7$, and $H_{0}=100 h \mathrm{~km} \mathrm{~s}^{-1} \mathrm{Mpc}^{-1}$. Absolute magnitudes are given for $h=1$.

\section{Data}

We use the spectroscopic catalogs of quasars and galaxies from the fifth (DR5) and sixth (DR6) Data Releases of the SDSS (Adelman-McCarthy et al. 2007). The original SDSS-DR5 galaxy sample contains 674749 galaxies, and the original quasar sample contains 90611 quasars. The DR6 contains 790220 galaxies. We use three different samples: volume-limited samples of galaxies from the DR5 to estimate the number densities of galaxies, volume-limited samples of groups of galaxies from the DR6 to study the group properties, and a magnitude-limited sample of galaxies to study the effects of environment on supercluster scales.

The final data for galaxies in this paper is taken from the Tago group catalog (Tago et al. 2008), based on the main galaxy sample obtained from the SDSS Data Archive Server, with 488725 galaxies. Applying the lower $(z=0.009)$ and upper $(z=0.12)$ redshift limits and excluding several duplicate galaxies in the original data, the number of galaxies was reduced to 387063 .

To study how nearby quasars are located among galaxies we extracted four volume-limited subsamples of galaxies with the absolute magnitude limits of $M_{\text {gal }}<-21.0, M_{\text {gal }}<-21.5$, $M_{\text {gal }}<-22.0$, and $M_{\text {gal }}<-22.5$ (Petrosian r-magnitudes). The samples contain 106378, 37181,7790 and 985 galaxies, respectively. For the limit $M_{\mathrm{gal}}<-21.0$ the comoving distance was selected to be less than $500 \mathrm{~h}^{-1} \mathrm{Mpc}$, while the lack of very nearby quasars imposed a lower distance limit of $200 \mathrm{~h}^{-1} \mathrm{Mpc}$. The value of $M^{*}(r)$ for galaxies in the SDSS is -20.44 , and therefore our sample consists of the brightest end of the luminosity function (Blanton et al. 2003).

The final group catalog that we use for our group analysis is a volume-limited sample based on the DR6 with the magnitudes $-22.0<M_{\text {group }}<-21.0$. This sample contains 9581 groups, and extends to the comoving distance of $450 \mathrm{~h}^{-1} \mathrm{Mpc}$. Groups in the SDSS DR6 were defined applying the well-known Friends-ofFriends cluster analysis method introduced by Turner \& Gott (1976), and modified by E. Saar. In this algorithm, galaxies are linked into systems using a variable linking length. By this definition, a galaxy belongs to a group of galaxies if this galaxy has at least one group-member galaxy closer than the linking length. In a flux-limited subsample, the linking length was increased with distance to keep virial properties, such as virial radius, maximum size in the sky projection, and velocity dispersion similar at all distances. In the volume-limited subsample used in our study, the linking length was constant inside one particular subsample, but was changed from subsample to subsample of various absolute magnitude limits in order to keep the virial properties similar in all volume limited subsamples.

Finally, we use the Tago et al. (2008) magnitude-limited sample of galaxies to determine the luminosity-density field. The limiting magnitudes of the complete spectroscopic sample of the SDSS catalog used here are 14.5 and 17.77 in r band (see Tago et al. 2008, for the description of the selection effects taken into account for this magnitude-limited galaxy sample).

As our final quasar data, we use the value-added quasar catalog based on the DR5 (Schneider et al. 2007), which contains 
77429 quasars. These quasars have absolute $i$ band magnitudes of $M_{i}<-22.0$, and have emission lines with line widths larger than $1000 \mathrm{~km} \mathrm{~s}^{-1}$. With these conditions the sample should contain only classical quasars, whereas the original SDSS quasar catalog also contained other kinds of active galaxies. Due to the limits of our galaxy and group catalogs, we include only quasars at redshifts $0.078<z<0.172$. We also require the quasars to satisfy the target-selection flag BEST, which indicates the results obtained using the latest photometric software for the highest quality data. These conditions limit our sample to 174 quasars.

\section{Results}

\subsection{Galaxies and quasars}

We study the spatial density of galaxies around quasars on different scales and using different limiting magnitudes for the galaxies. As a control sample, we use the same galaxy catalogs used to measure the galaxy density.

As a first simple measure of the density, we estimate the redshift-space distance from each quasar to the nearest galaxy brighter than $M=-21.5$. The average distance between a quasar and its nearest galaxy is $(4.9 \pm 0.3) h^{-1} \mathrm{Mpc}$, while the average distance between a $M<-21.5$ galaxy and the nearest other galaxy is $(4.30 \pm 0.02) h^{-1} \mathrm{Mpc}$. Although this estimate is quite uncertain, it may be a hint of quasars lying in less dense regions than galaxies with $M<-21.5$ in general.

We then estimate the number density of galaxies at different distances from the quasars. We count the number of galaxies in $0.1 h^{-1} \mathrm{Mpc}$ bins from 0.1 to $4 h^{-1} \mathrm{Mpc}$ distance from each of the quasars. This number is then divided by the total comoving volume of this bin for all the quasars,

$$
\frac{\mathrm{d} N}{\mathrm{~d} V}\left(R_{i}\right)=\frac{\frac{1}{n} \sum_{j=1}^{n} N_{j}}{V\left(R_{i}\right)-V\left(R_{i-1}\right)}
$$

where $n$ is the number of quasars, $N_{j}$ is the number of galaxies at a distance $R_{i}$ from each quasar, and $V\left(R_{i}\right)$ is the comoving volume of a sphere with the radius $R_{i}$. This provides the number density of galaxies in this distance bin for all the quasars. The same calculation is completed for the reference galaxies.

Figure 1 shows the mean number density of galaxies as a function of distance from quasars and galaxies. The results provide a hint of a galaxy overdensity at radii of less than $1 h^{-1} \mathrm{Mpc}$ around quasars. However, because of the small number of quasars in our sample the Poissonian errors at the radii $<4 h^{-1} \mathrm{Mpc}$ are large, and the differences between quasars and galaxies are within the error limits.

To study the galaxy density inside the whole comoving volume enclosed by the $4 h^{-1}$ Mpc radius from the quasars, we measure the number distribution of galaxies inside this radius. The results for the environments of quasars and galaxies for the magnitude limits $M<-21.0$ and $M<-21.5$, respectively, are shown in Fig. 2. Of the quasars in our sample, $28 \%$ have no $M<-21.0$ galaxies inside the $4 h^{-1}$ Mpc radius, while for the $M<-21.0$ galaxies themselves, this fraction is only $21 \%$. The Kolmogorov-Smirnov test (Table 1) shows that the distributions are different on the $98.6 \%$ significance level. This suggests that the quasars reside in poorer environments than the $M<-21.0$ galaxies. Differences in the distributions for the limiting galaxy magnitude $M<-21.5$ are less significant, but Fig. 2 shows that the fraction of quasars with no galaxies within the $4 h^{-1} \mathrm{Mpc}$ radius is slightly higher than the fraction of galaxies. For magnitude limits $M<-22.0$ and $M<-22.5$, the number of galaxies is too small to show any differences.
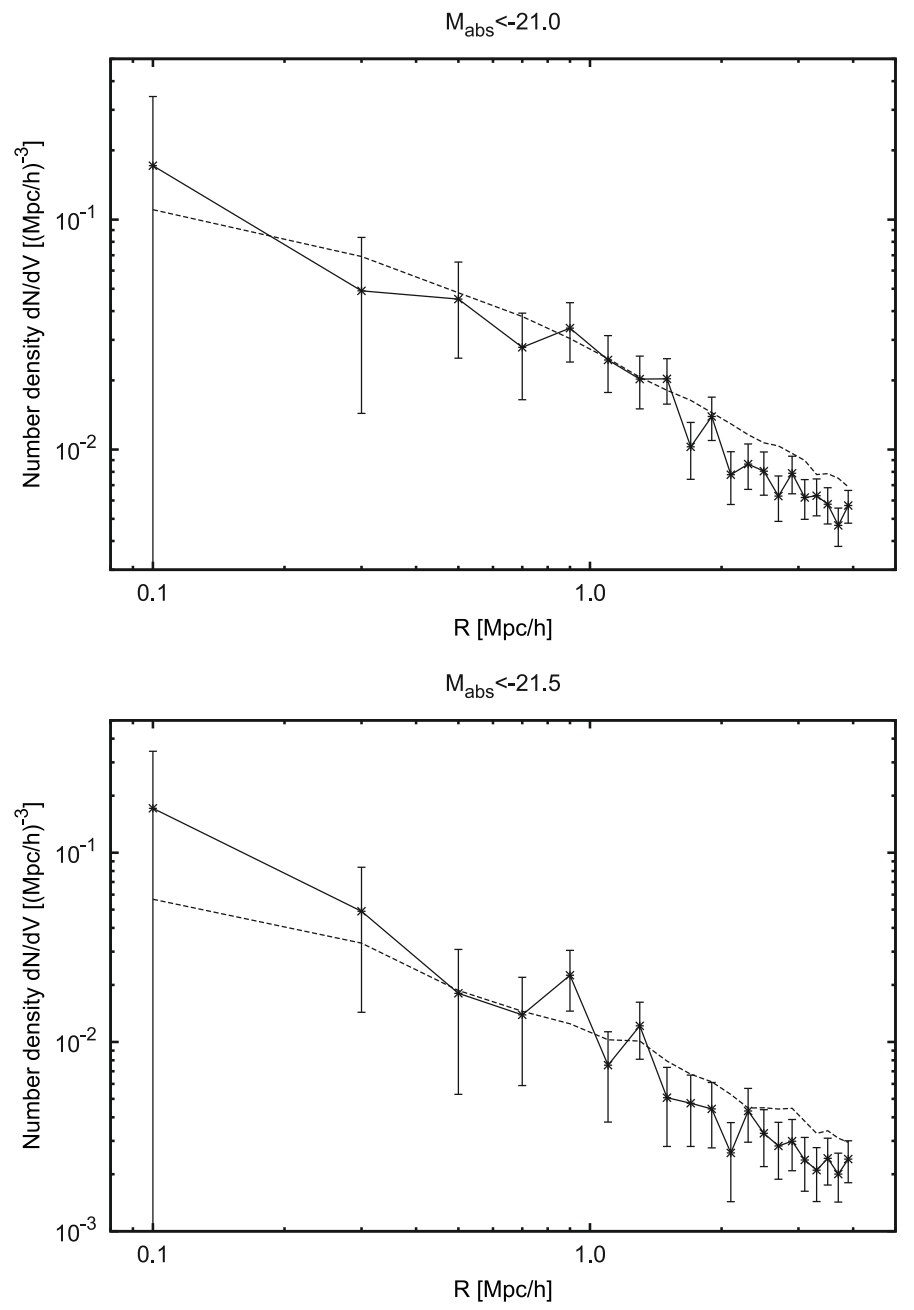

Fig. 1. The spatial density of galaxies at radii $r<4 h^{-1} \mathrm{Mpc}$ around quasars (solid line, with error bars) and galaxies (dashed line) for the galaxy luminosity limits $M<-21.0$ (top) and $M<-21.5$ (bottom).

Table 1. Kolmogorov-Smirnov test statistics for distributions of the number of galaxies inside 4 and $10 h^{-1} \mathrm{Mpc}$ radii around quasars and galaxies.

\begin{tabular}{cccc}
\hline \hline Magnitude Limit & Distance Limit $\left[h^{-1} \mathrm{Mpc}\right]$ & $\hat{D}$ & Probability \\
\hline-21.0 & 4 & 0.118 & 0.01442 \\
-21.5 & 4 & 0.086 & 0.1467 \\
-21.0 & 10 & 0.141 & $1.748 \times 10^{-3}$ \\
-21.5 & 10 & 0.139 & $2.176 \times 10^{-3}$ \\
-22.0 & 10 & 0.203 & $1.227 \times 10^{-6}$ \\
-22.5 & 10 & 0.197 & $1.621 \times 10^{-5}$ \\
\hline
\end{tabular}

Probabilities for the distributions to be similar are shown in the fourth column.

Figure 3 shows the spatial density of galaxies around quasars on larger scales, from $2 h^{-1} \mathrm{Mpc}$ to $30 \mathrm{~h}^{-1} \mathrm{Mpc}$. This was calculated in the same way as the galaxy density on smaller scales, but the bin is now $4 h^{-1} \mathrm{Mpc}$. Here, we use two more magnitude limits for galaxies, $M<-22.0$ and $M<-22.5$. On this scale, quasars are located in less dense regions than galaxies on average. When the galaxy luminosity limit increases from $M<-21.0$ to $M<-22.5$, the difference between the quasar and galaxy environments increases.

We also calculated the number densities for a volume-limited sample of galaxies with $M<-20.0$. This sample extends only 

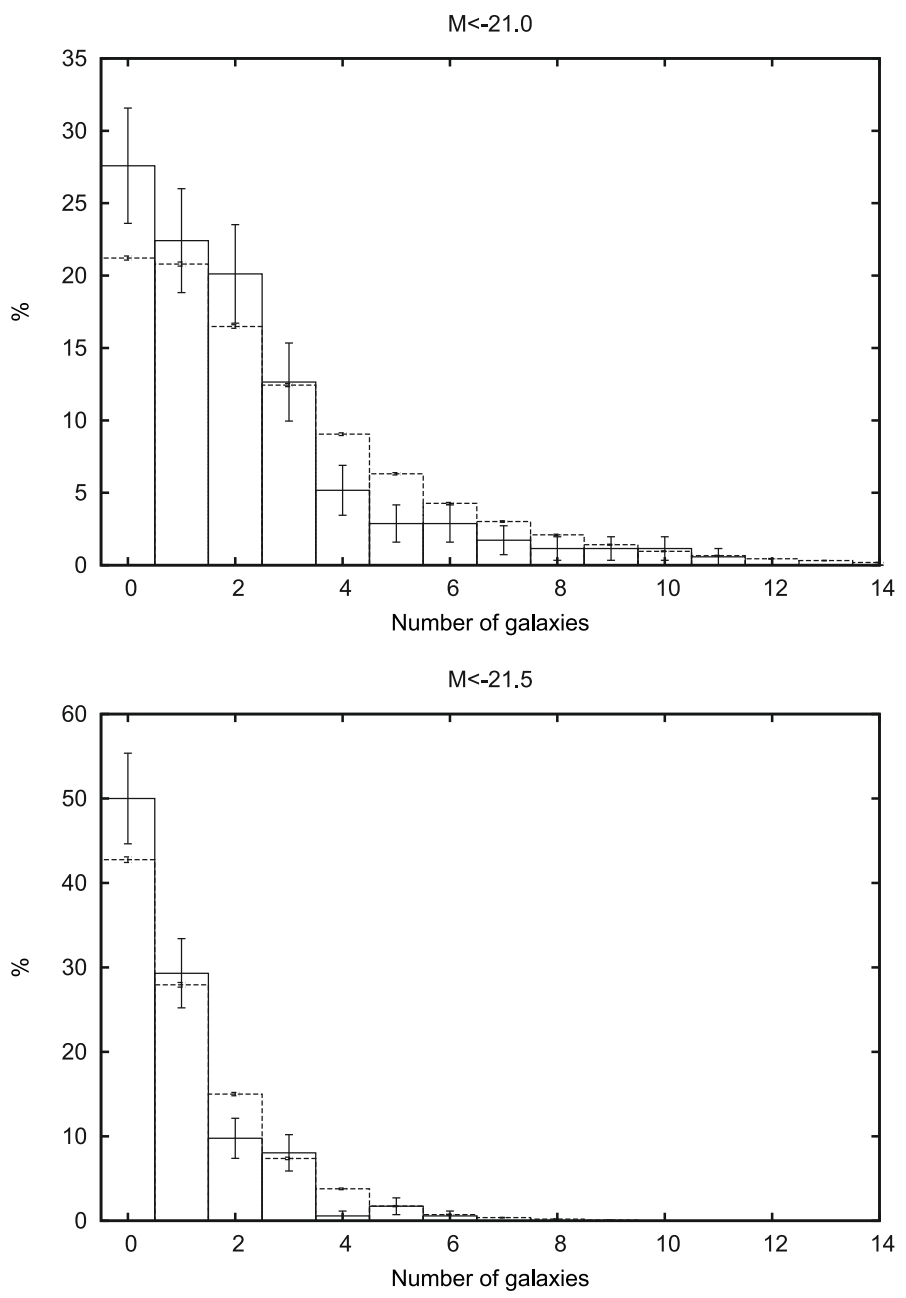

Fig. 2. Distribution of the number of galaxies inside $4 h^{-1} \mathrm{Mpc}$ radii from quasars (solid line histogram) and galaxies (dashed line histogram) with limiting magnitudes of $M<-21.0$ (top) and $M<-21.5$ (bottom) for the galaxies.

to $316 h^{-1} \mathrm{Mpc}$, which limits the number of quasars to 11 . An underdensity around quasars can be detected, but there are insufficient data to give accurate results.

The underdensity around quasars can also be seen in Fig. 4, which shows that the number of galaxies closer than $10 \mathrm{~h}^{-1} \mathrm{Mpc}$ to quasars tends to be lower than the number of other galaxies closer than $10 h^{-1} \mathrm{Mpc}$ to galaxies. For $M<-21.0$ galaxies, $45 \%$ of quasars have fewer than ten galaxies within the $10 h^{-1}$ Mpc distance, while of the $M<-21.0$ galaxies themselves, only $33 \%$ belong to this group. The effect is stronger for brighter galaxies. For the magnitude limit $M<-22.0,50 \%$ of quasars have no galaxies within the $10 \mathrm{~h}^{-1} \mathrm{Mpc}$ distance, while $30 \%$ of the $M<-22.0$ galaxies have no other galaxies with the same magnitude limit closer than $10 h^{-1} \mathrm{Mpc}$. As we can see in Table 1, the distributions at all the magnitude limits are different at very high levels of significance.

We also completed number density analysis for a smaller subsample of the galaxy catalog in order to estimate the effect of the edges of the sample volume. We chose 15 subsamples from the galaxy catalog with the limiting magnitude $M<-21.5$, such that all the galaxies in the subsamples lie at least $60 \mathrm{~h}^{-1} \mathrm{Mpc}$ from the edges of the full sample. The number densities of these galaxies compared to the whole galaxy sample is shown in Fig. 5. This analysis showed us that the estimate of the number density is not significantly biased by the edges of the sample for distances smaller than $20 h^{-1} \mathrm{Mpc}$. For the neighbor distance $50 \mathrm{~h}^{-1} \mathrm{Mpc}$, our estimate is approximately $30 \%$ too small.

\subsection{Groups and quasars}

Our volume-limited group catalog contains 9581 groups, but a vast majority are groups of only two galaxies. In some cases, we use a subsample of the 777 groups that have 4 or more galaxies. In this way, we can see whether the properties of rich groups near quasars differ from the properties of poor groups.

We study the distribution of groups of galaxies in the environments of quasars in a similar manner as we did for the distribution of galaxies. The average distance from a quasar to the nearest group is $(7.8 \pm 0.5) h^{-1} \mathrm{Mpc}$ and the average distance from a galaxy with $M<-21.5$ to the nearest group is $(6.49 \pm 0.04) h^{-1} \mathrm{Mpc}$. This result suggests that quasars are farther away from the groups than galaxies on average. However, if we compare only the distances to the richer groups, we find no difference at all: the average distance from a quasar to the nearest group of at least 4 galaxies is $(22 \pm 2) h^{-1} \mathrm{Mpc}$, while the average distance from a $M<-21.5$ galaxy to the nearest rich group is $(21.64 \pm 0.08) h^{-1} \mathrm{Mpc}$.

The average luminosities and numbers of galaxies of groups that have quasar or galaxy neighbors at a given distance are shown in Fig. 6. The galaxy sample used for comparison has the limiting magnitude $M<-21.0$. The plots in the top part of Fig. 6 are cumulative; e.g., the values at $R=5 h^{-1} \mathrm{Mpc}$ are the averages for all the groups that have at least one quasar or galaxy neigbor closer than $5 h^{-1} \mathrm{Mpc}$. The mean luminosity of all the groups in the sample is $5.64 \times 10^{10} L_{\odot}$, and the mean number of galaxies in the groups is 2.38 . Both curves approach the average for all groups as the neighbor distance increases.

Figure 6 suggests that the groups that have a quasar at a distance less than $2 h^{-1} \mathrm{Mpc}$ have very low luminosities and a small numbers of galaxies. This result is uncertain because the number of these groups is small, only 14 groups have quasars at $R<2 h^{-1} \mathrm{Mpc}$. Another feature that can be seen in the figure is that the groups that have quasar neighbors at distances of 10 to $15 h^{-1} \mathrm{Mpc}$ are brighter and richer than the groups that have bright galaxy neighbors at the same distances. This can also be seen in the differential plot, shown in the bottom panels of Fig. 6: the richest and most luminous groups are the ones that have a quasar neighbor at distances from 5 to $15 h^{-1} \mathrm{Mpc}$. However, the differences are small, and because of the small number of quasars, it is uncertain wheter the correlations are real.

In groups of only two or three galaxies, the velocity dispersion does not provide reliable information about the physical nature of the group. If we consider the subsample of rich groups of four or more galaxies, we can study dynamical properties of the groups. Figure 7 shows the average luminosities, numbers of galaxies, and rms velocities for the rich groups that have quasar neighbors closer than $R$.

If we consider only the rich groups, we see the same increase in the luminosity and the number of galaxies as we saw in the case of all the groups that have quasar neighbors closer than $10 h^{-1} \mathrm{Mpc}$. The rich groups that are very close to a quasar are clearly poorer and less luminous than the rich groups in general. The richest and most luminous groups have their closest quasar at approximately $10-15 h^{-1} \mathrm{Mpc}$ from the group. This effect can also be seen in the rms velocities, although it is not as clear as for the luminosity and number of galaxies.

The rise in group richness and luminosity at the 10$15 h^{-1}$ Mpc distance from quasars could be explained by 

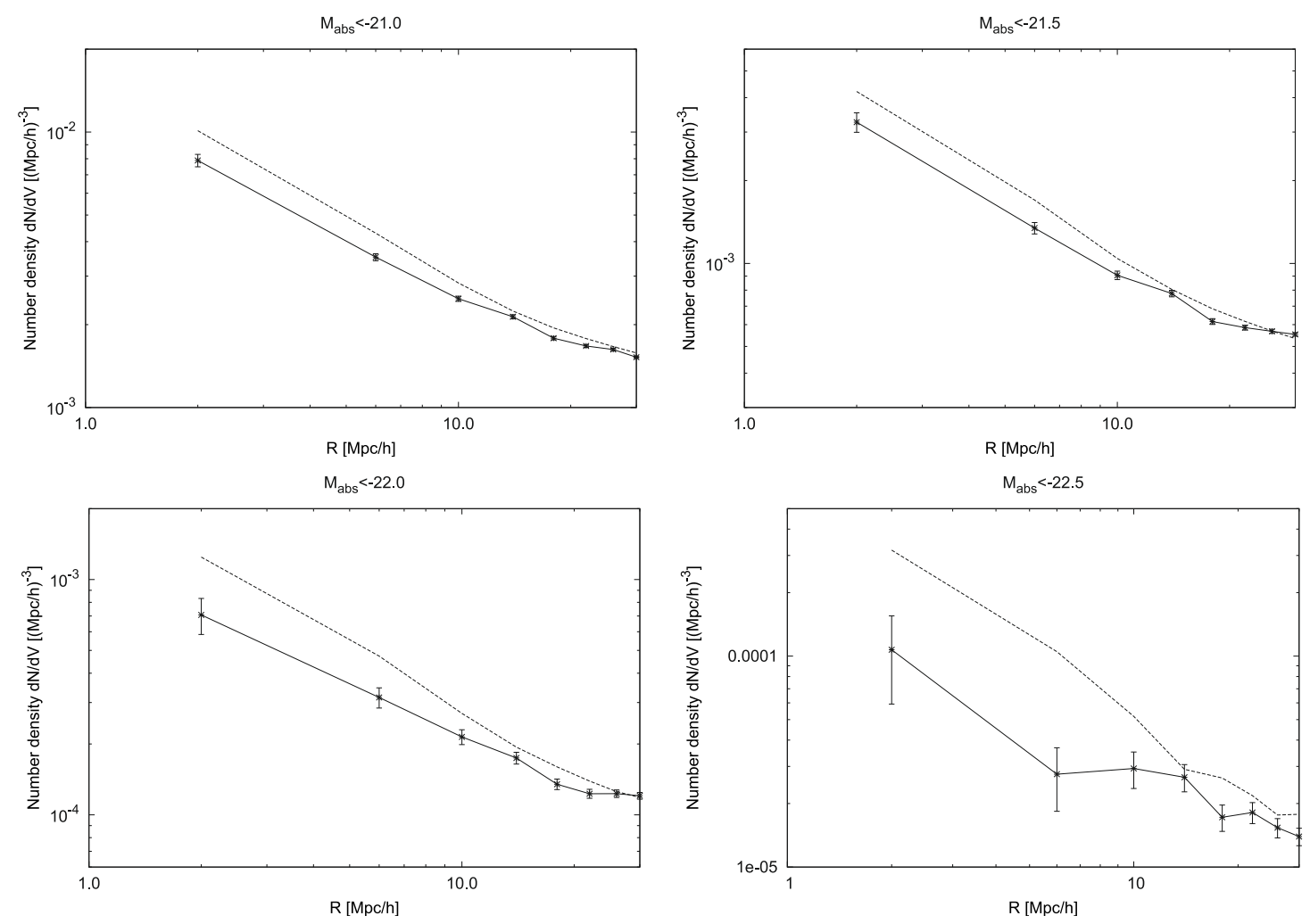

Fig. 3. The spatial density of galaxies at radii $r<30 h^{-1} \mathrm{Mpc}$ around quasars (solid line) and galaxies (dashed line) for galaxy luminosity limits $M<-21.0, M<-21.5, M<-22.0$, and $M<-22.5$.
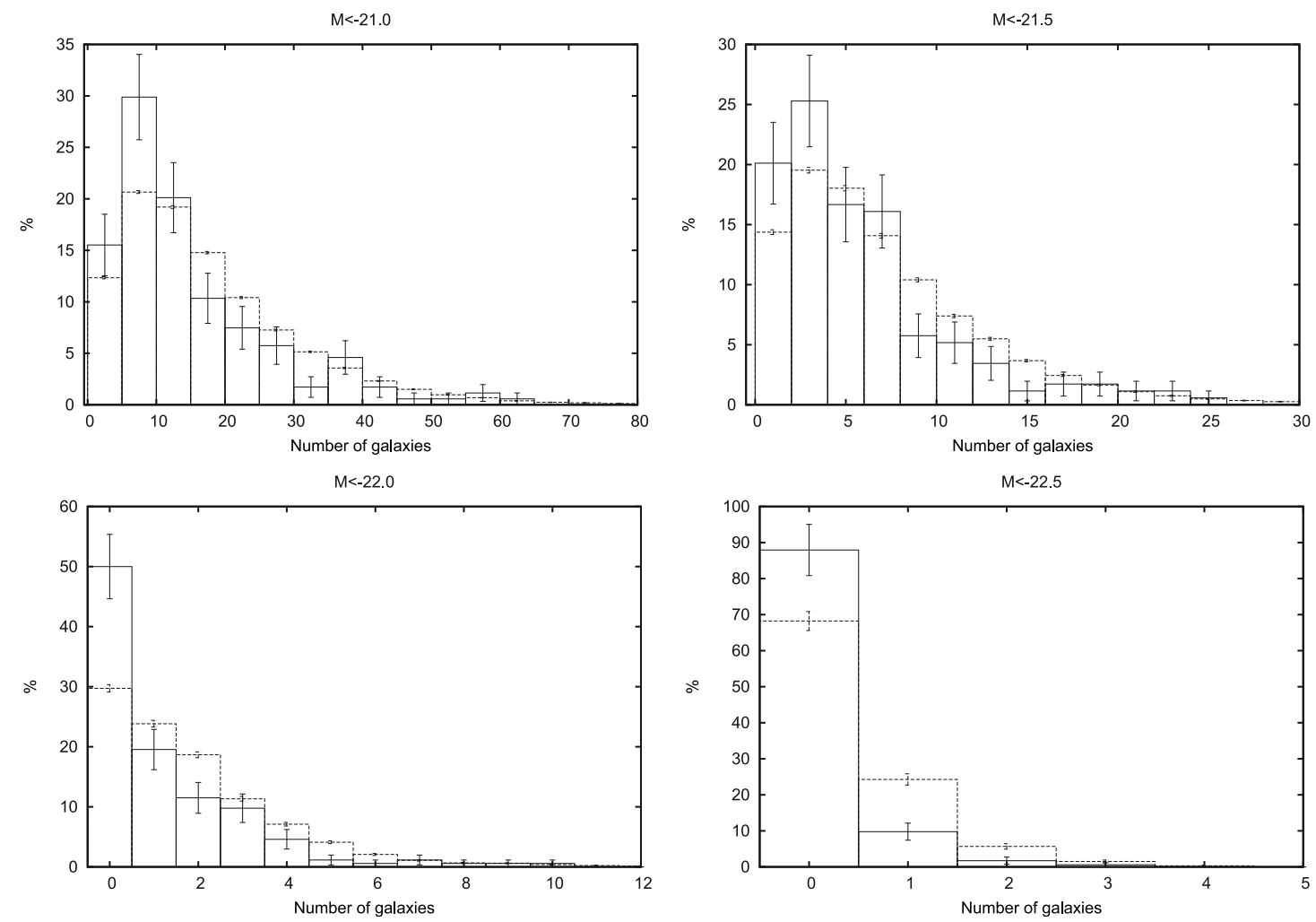

Fig. 4. Distribution of the number of galaxies inside $10 \mathrm{~h}^{-1} \mathrm{Mpc}$ radii from quasars (solid line histogram) and galaxies (dashed line histogram) with limiting magnitudes for galaxies $M<-21.0$ (top left panel), $M<-21.5$ (top right), $M<-22.0$ (bottom left), and $M<-22.5$ (bottom right). 


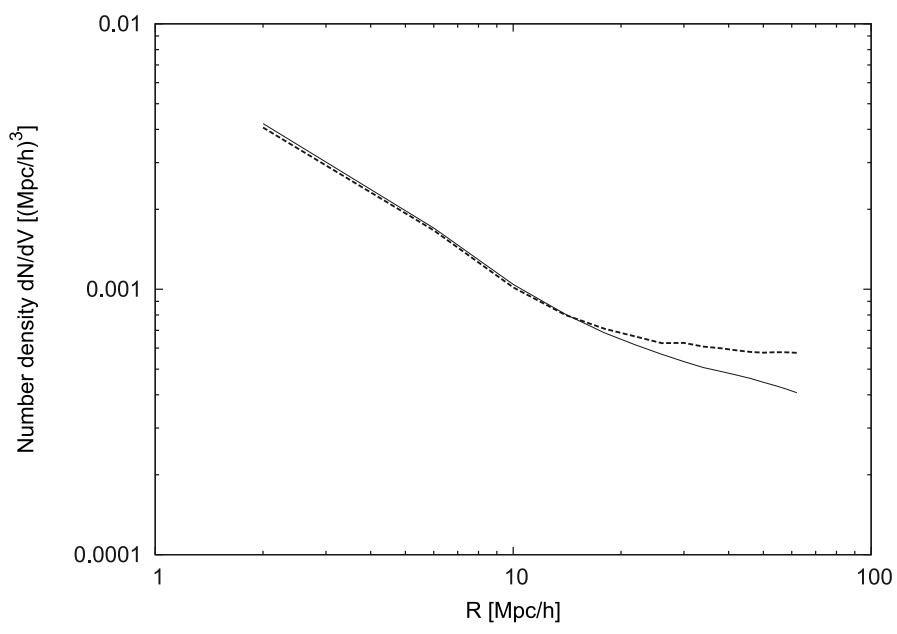

Fig. 5. Edge effects in the galaxy samples. Number density of all galaxies is shown with the solid line, and number density of galaxies in the 15 subsamples with the minimum distance to edges of the whole galaxy sample of $60 h^{-1} \mathrm{Mpc}$ or more with the dashed line.

the distribution of quasars within the large-scale structure. The richest groups usually lie at the cores of superclusters, and $10 h^{-1} \mathrm{Mpc}$ is a typical distance between a core and a filament (Einasto 2007b). Therefore, this result could be explained by quasars being located preferentially in filaments.

\subsection{Superclusters and quasars}

The most significant density enhancements in the Universe, superclusters and their environments, can be studied using the luminosity-density field of galaxies (Einasto et al. 2003). It has been shown that the morphological properties of galaxies depend on their large-scale cosmological environment (Einasto et al. $2007 \mathrm{a}$, and references therein). Both the local (group or cluster scale) and global (supercluster scale) environments influence galaxy morphology and their star-formation activity. Rich superclusters contain a higher fraction of early-type, passive, red galaxies than poor superclusters (Einasto et al. 2007b). Inside rich superclusters galaxy properties also vary. The core regions of rich superclusters contain a higher fraction of early-type, red galaxies and richer groups than the outskirts of superclusters (Einasto et al. 2008). Galaxy clusters in high-density environments are about 5 times more luminous than in low-density environments (Einasto et al. 2005).

The luminosity-density field smoothed to appropriate scales allows us to study the locations of nearby quasars in the supercluster-void network. Voids, filaments, and superclusters have characteristic density levels within the overall large-scale structure; thus, the density level can be used to discriminate between different details of the network. In this procedure, we use all the SDSS DR5 galaxies from the Tago catalog of groups and isolated galaxies (Tago et al. 2008). This catalog is suitable for tracing the supercluster-void network; groups are mostly density enhancements within filaments and rich clusters are high density peaks of the galaxy distribution in superclusters (Tago et al. 2008, and references therein).

We assume that every galaxy is a visible member of a density enhancement (a group or a cluster). We correct the galaxy luminosities by a weighting factor that accounts for the expected group galaxies outside the visibility window:

$L_{\mathrm{tot}}=L_{\mathrm{obs}} W_{L}$, where $L_{\mathrm{obs}}$ is the luminosity of an observed galaxy and $W_{L}$ is the ratio of the total luminosity to the luminosity in the visibility window $L \in\left[L_{1}, L_{2}\right]$ :

$W_{L}=\frac{\int_{0}^{\infty} L \phi(L) \mathrm{d} L}{\int_{L_{1}}^{L_{2}} L \phi(L) \mathrm{d} L} ;$

$\phi(L)$ is the galaxy luminosity function. According to Fig. 11 in Tago et al. (2008), the parameter $W_{L}$ is less than two at the distances from 100 to $300 \mathrm{~h}^{-1} \mathrm{Mpc}$, and increases to 5 at $500 h^{-1} \mathrm{Mpc}$. Finally, group and cluster galaxies are positioned at the mean group-cluster distance, to correct for the effect of the dynamical velocities (redshift space fingers).

A Cartesian grid is then defined for the survey volume and the luminosity-density field on the grid is calculated using the B3-spline kernel function. Each galaxy contributes its luminosity to the density field. This gives us the total luminosity-density field of the survey where different characteristic structures have different density levels.

The total luminosity in a subvolume can be calculated by summing over all the grid vertices it contains (the luminosity density units are $1.0 \times 10^{10} /\left(D^{3}\right)\left[L_{\odot} /\left(h^{-1} \mathrm{Mpc}\right)^{3}\right]$, where $D$ is the grid cell size in $\left.h^{-1} \mathrm{Mpc}\right)$. Using an appropriate mass-toluminosity ratio, one can translate luminosities into masses. We chose the cell size of $1 h^{-1} \mathrm{Mpc}$, since this is the characteristic size of a cluster. The half-width of the B3 smoothing kernel was $16 h^{-1} \mathrm{Mpc}$, and the effective smoothing radius (the kernel halfwidth for which the density value differs considerably from zero) is $8 h^{-1} \mathrm{Mpc}$. This is an appropriate value for characterizing the global environment and superclusters (Einasto et al. 2007a).

Regions of luminosity density $D_{L}<1.5$ (in units of the mean luminosity-density) are considered to be voids. Superclusters are systems that occupy regions above the threshold density of about $D_{L}>4.6$ (Einasto et al. 2007a). This is approximately the lowest limit for separating non-percolating galaxy systems (poor superclusters) from the overall density field. Densities that correspond to rich superclusters have a median value of $D_{L}=7.5$ times the mean density and $D_{L}>10$ correspond to cores of rich superclusters (Einasto et al. 2007b). These values were determined for $2 \mathrm{dFGRS}$ data, and there may be some differences for the superclusters in the SDSS. However, the differences should not be large enough to influence our analysis.

Figure 8 shows the projected luminosity density field of the SDSS DR5 northern and equatorial samples. Red regions show the most luminous centers of the superclusters with densities of about 20 times the mean luminosity density. The circles in the figure represent quasars. As we can see, quasars seem to be located in low density regions, on the outskirts of superclusters.

A more detailed analysis reveals that the local mean luminosity-density around quasars is $D_{L} \approx 2.4$. This is illustrated in Fig. 9, which shows the distribution of the mean luminosity density at quasar locations (in a sphere of radius of $2 h^{-1} \mathrm{Mpc}$ ) - the number of quasars with a given environmental density.

Most of the quasars seem to be located in a low-density environment on these scales. Quasars avoid densities that are characteristic of poor superclusters, $D_{L}>4.6$, and none is found in high density environments, $D_{L}>10$.

Figure 10 shows the mean luminosity density of galaxies in north and equatorial samples around quasars as a function of distance. Two different threshold densities, $D_{L}>4.6$ and $D_{L}>10$ are used to separate poor and rich superclusters. As we can see, regions with $D_{L}>4.6$ belong to the nearby quasar environments. When the threshold value is increased to $D_{L}>10$, no 

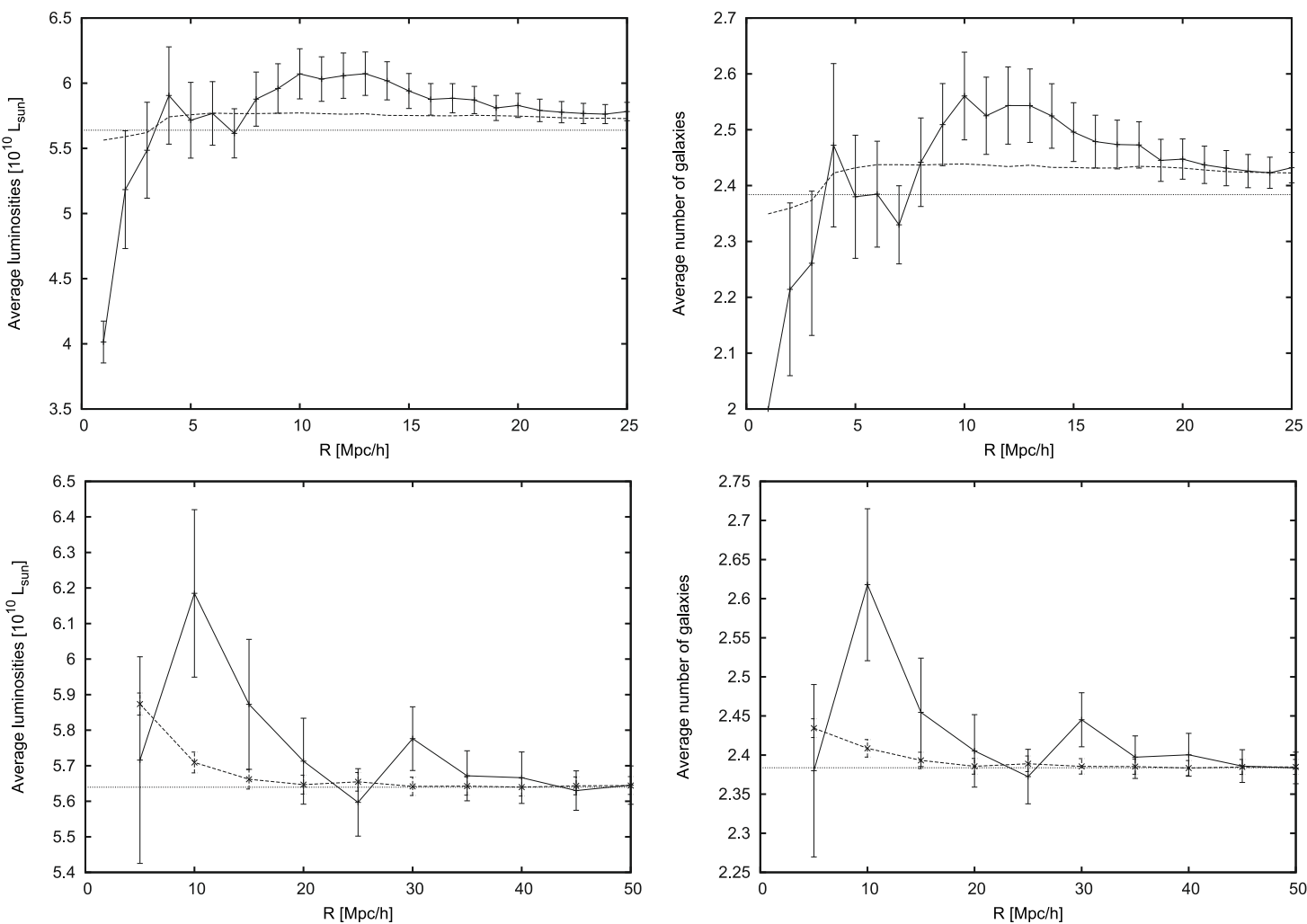

Fig. 6. Bottom: average luminosities (left) and numbers of galaxies (right) of groups that have quasar neighbors (solid line) or galaxy neighbors (dashed line) between the radii $R-5 h^{-1} \mathrm{Mpc}$ and $R$. The average luminosity and number of galaxies of all groups in the sample are shown with dotted lines. Top: average luminosities (left) and numbers of galaxies (right) of groups that have quasar neighbors (solid line) or galaxy neighbors (dashed line) inside the radius $R$. The average luminosity and number of galaxies of all groups in the sample are shown with dotted lines.
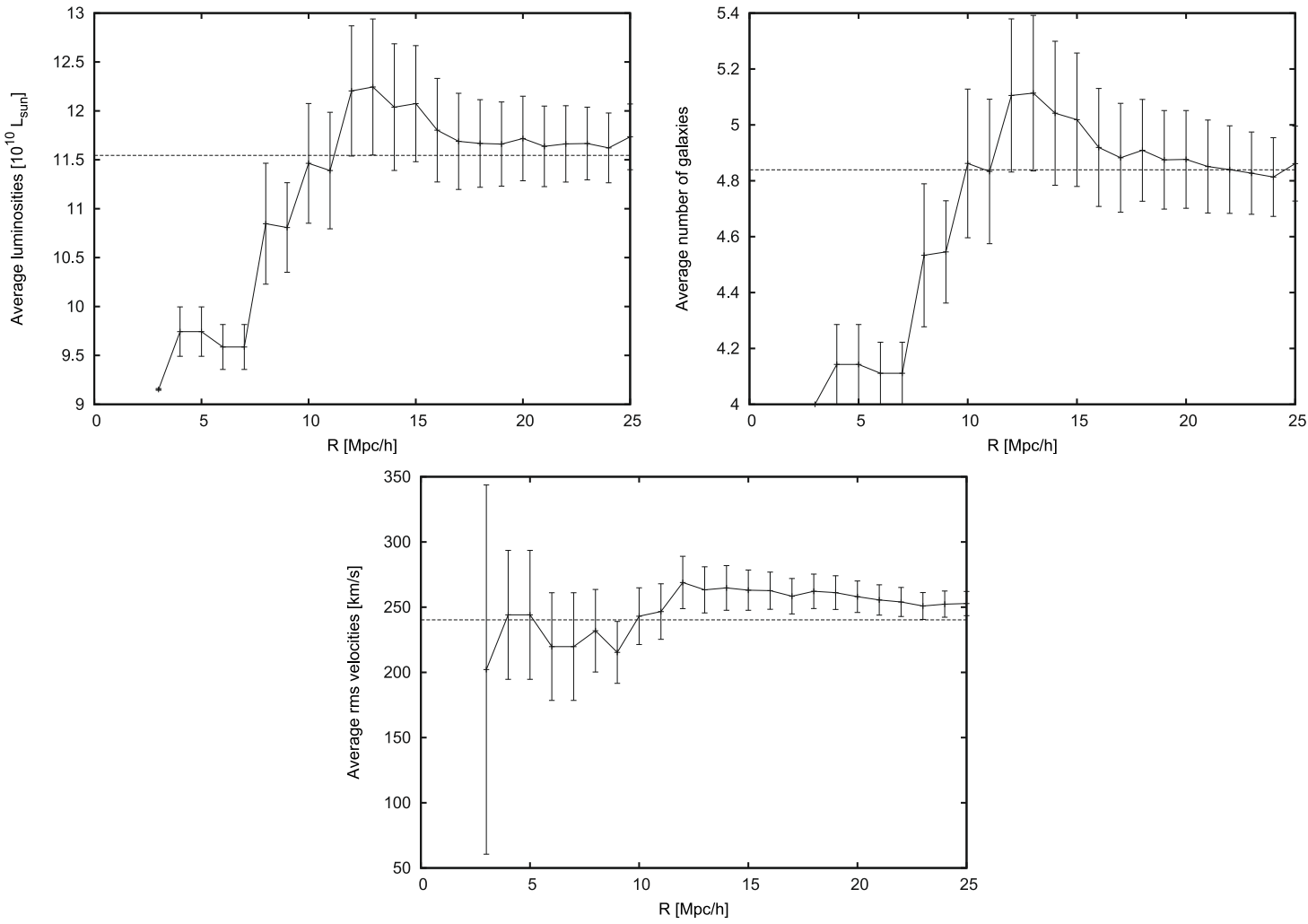

Fig. 7. Average luminosities (top left), numbers of galaxies (top right), and rms velocities (bottom) of groups of at least 4 galaxies that have quasar neighbors inside the radius $R$. The averages for all rich groups in the sample are shown with dashed lines. 

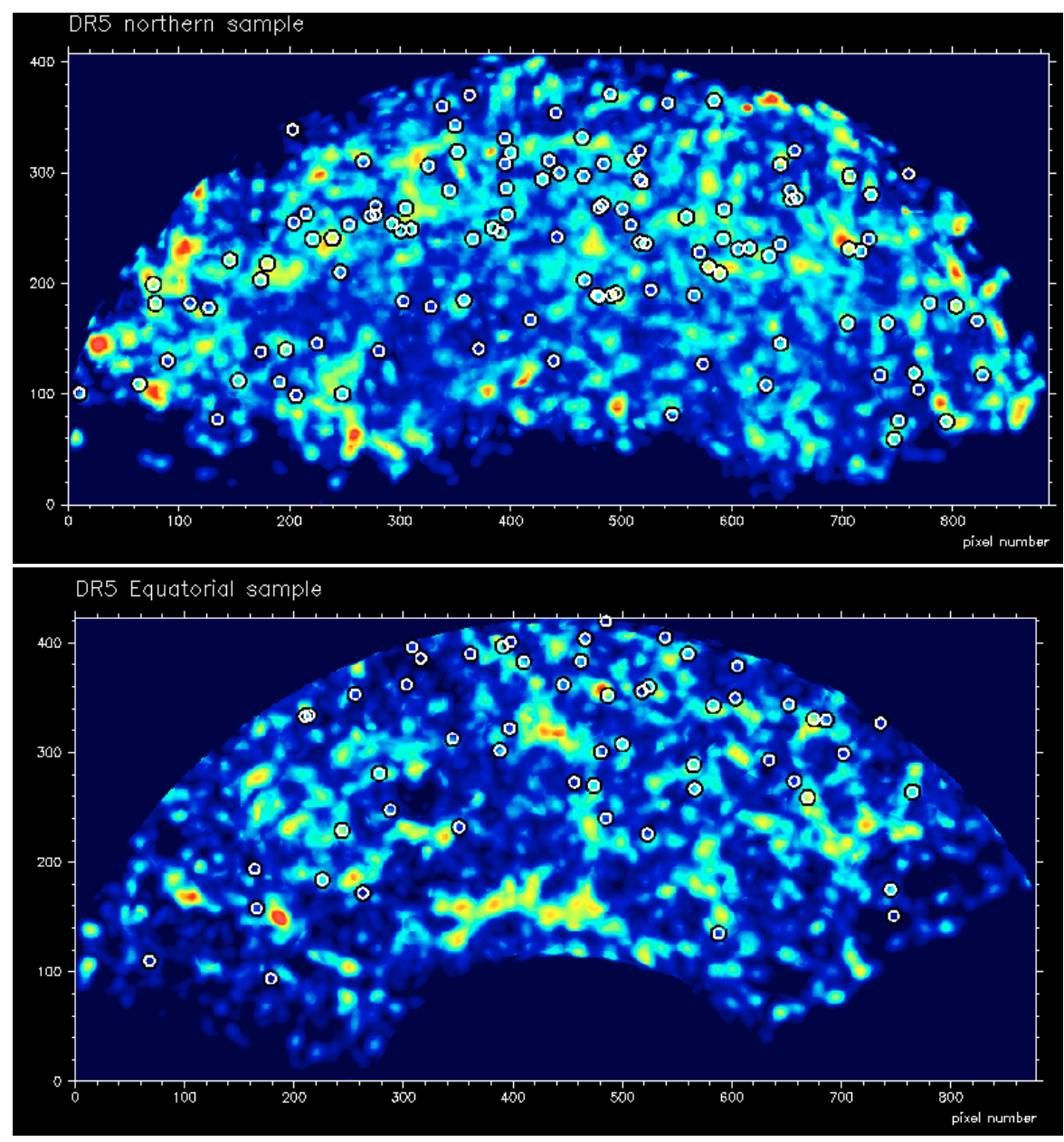

Fig. 8. Quasars in the luminosity density field of the northern sample (top) and the equatorial sample (bottom) of the SDSS. (A color version of this figure is available in the online journal.)

nearby quasars are found. If we increase the threshold value even higher, the high-density regions are located mostly at distance of approximately $10 \mathrm{~h}^{-1} \mathrm{Mpc}$ from the quasars. This is close to the distance found for the analysis based on the volume-limited galaxy sample in Sect. 3.2. This scale agrees with the characteristic scale for superclusters and void sizes found in observations and simulations (von Benda-Beckmann et al. 2008; Ceccarelli et al. 2004; Gottlöber et al. 2003).

This characteristic distance is also clearly evident in Fig. 11, which shows the $D_{L}$ values for the full luminosity-density grid versus the distance to the nearest quasar. In both panels of Fig. 11, we see the highest luminosity densities for distances of about $10 h^{-1} \mathrm{Mpc}$ from quasars. Differences between the northern and equatorial samples in Figs. 10 and 11 are most probably caused by quantitative and intrinsic variance in the relatively large and complex filamentary structures of the superclusters between two samples of different sizes. The volume of the equatorial sample is about half of the northern one. Differences between these samples are also visible in Fig. 8. This analysis indicates that nearby quasars avoid high-density regions and prefer poor superclusters, outskirts of rich superclusters, and filaments.

The most luminous galaxies as well as the richest groups are located in the core regions of rich superclusters (Einasto et al. 2007b). This is in accordance with the findings that quasars avoid rich groups and prefer filaments close to superclusters. Thus our luminosity density analysis agrees with our result in

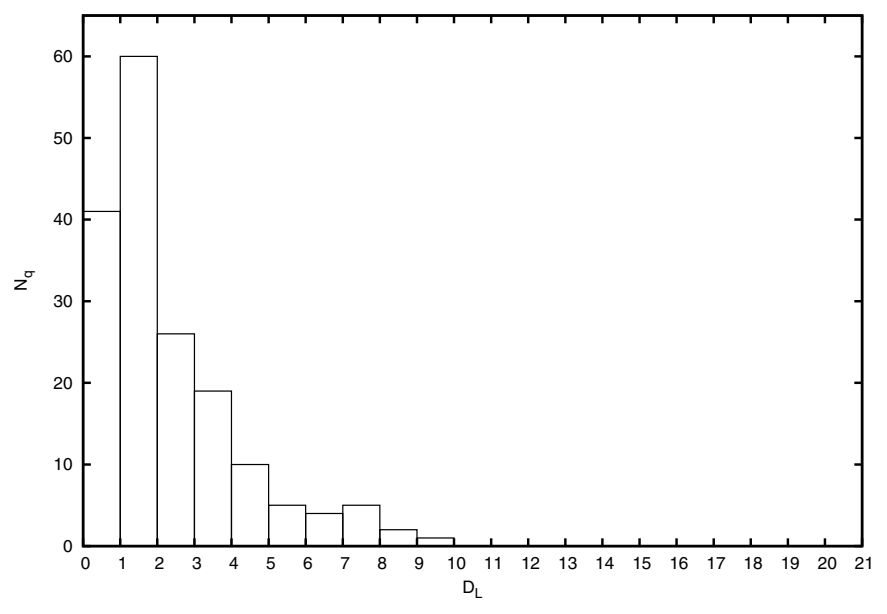

Fig. 9. The number of nearby quasars with a given environmental luminosity density $D_{L}$ (in a ball of the radius of $2 h^{-1} \mathrm{Mpc}$ ).

Sects. 3.1 and 3.2, where we showed that the galaxy number density around QSOs is a function of the luminosity of galaxies.

\section{Discussion}

One of our main results is that nearby quasars are located in lowdensity environments among luminous galaxies on a few Mpc 

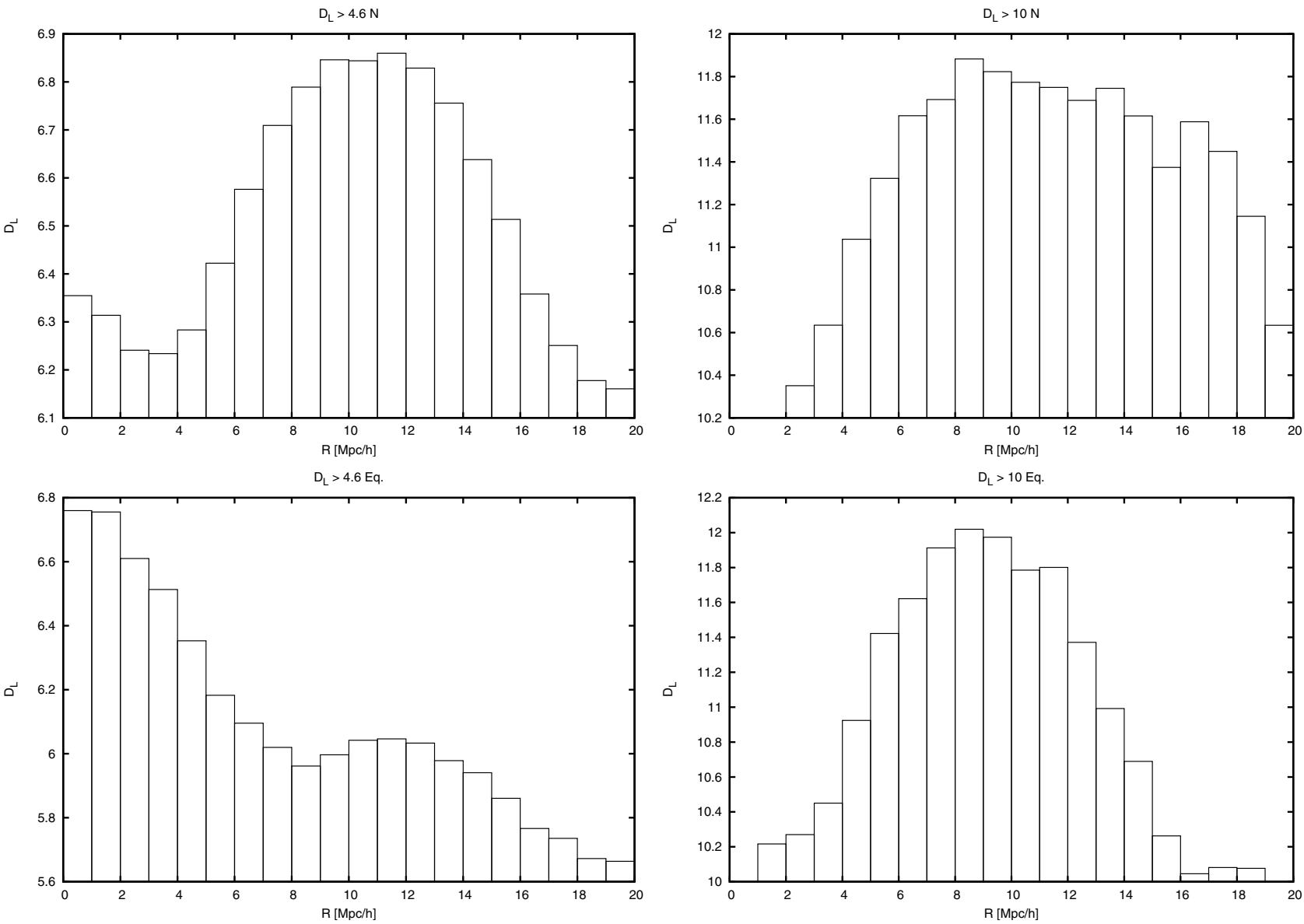

Fig. 10. The mean luminosity density of galaxies in the north $(\mathrm{N})$ and equatorial (Eq.) SDSS-DR5 volumes as a function of distance from quasars. The density thresholds are $D_{L}>4.6$ and $D_{L}>10$.
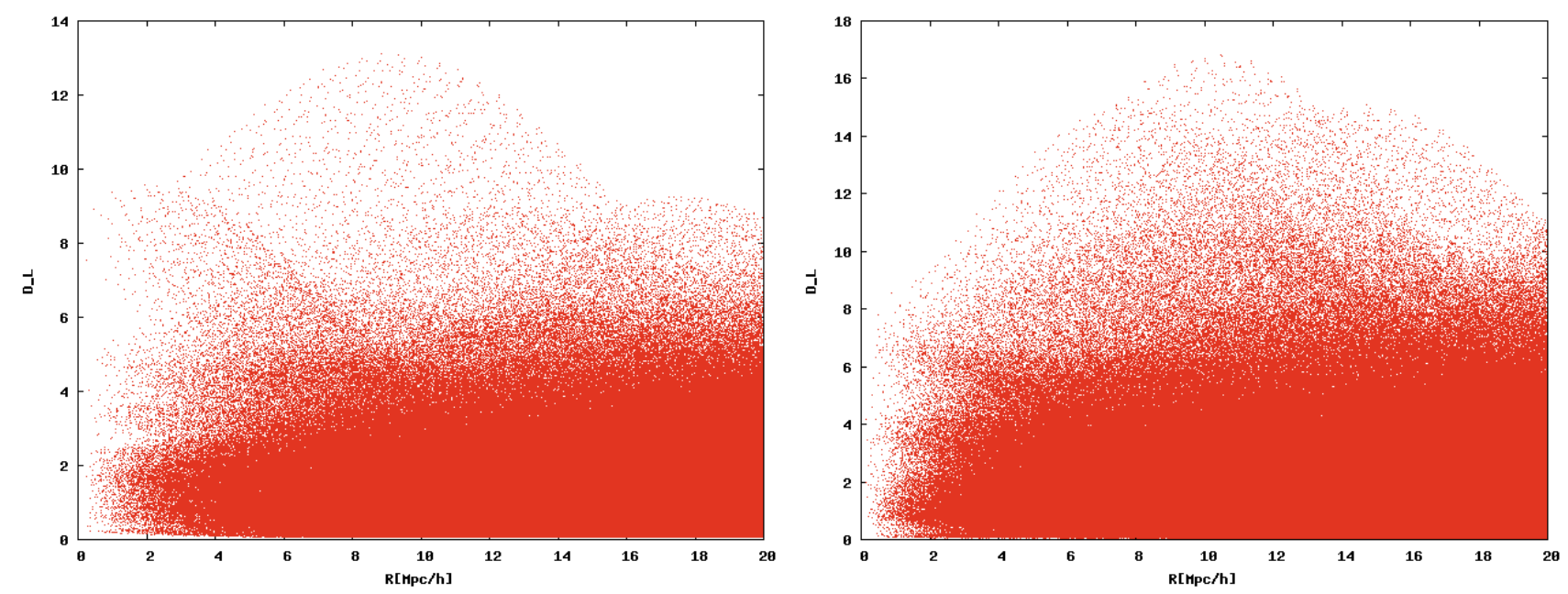

Fig. 11. All luminosity density values in the north (right) and equatorial (left) SDSS-DR5 volumes as a function of distance from quasars.

scale. This confirms the result of Coldwell \& Lambas (2006) for quasars in the same redshift range as studied here. Strand et al. (2008) found that quasars are located in more dense regions than other types of active galaxies. They studied environments of quasars on scales $<2 \mathrm{Mpc}$, while we have analyzed mostly larger scales. Moreover, we have studied only the distribution of bright galaxies; the density of fainter galaxies in the close environments of quasars could be higher.
Several studies have demonstrated that the properties of galaxies and dark matter halos depend on their environments in several ways (Kauffmann et al. 2004; Harker et al. 2006; Balogh et al. 2004; Tempel et al. 2008). For example, Constantin et al. (2008) stated that void and cluster galaxies follow different evolutionary paths, a result that which should also apply to active galactic nuclei. Presumably, the accretion process of the supermassive black hole (SMBH) in a galaxy center is also different for different environments. 
An observationally well-justified relation exists between the masses of the SMBH and the bulge velocity dispersion of the host galaxy (e.g, Magorrian et al. 1998; Graham et al. 2001; Tremaine et al. 2002). McLure \& Dunlop (2004) applied this relation in estimating the $\mathrm{BH}$ masses of 12698 quasars in the first release of the SDSS, and found that quasar SMBH masses are between $\sim 10^{7} M_{\odot}$ and $\sim 10^{9} M_{\odot}$. The connection between the growth of the black holes and the underlying structure formation was shown by Ferrarese (2002). She pointed out that the mass of the SMBH correlates with the mass of the dark matter halo in which they were presumably formed. Combining the results on quasar-mass limits by McLure \& Dunlop (2004) with the results of Ferrarese (2002; Fig. 5 and Eq. (6) in her paper), we can estimate that the masses of the dark matter host halos of the local quasars should have been around $10^{12}$ to $10^{13} M_{\odot}$. These mass limits are also compatible with the results of Croom et al. (2005), which suggested that the mass of the dark matter halo of a quasar is more or less independent of the redshift. This means that the present-day host dark matter halos of the nearby quasars should have the same mass range of $10^{12}-10^{13} M_{\odot}$.

Using simulations, Gao et al. (2005) showed that, on large scales, dark matter halo-clustering depends on the age of the halo. They concluded that halos of a given mass that assembled at high redshift are substantially more strongly clustered than halos of the same mass that assembled recently. The "oldest" 10 per cent of halos are more than five times more strongly correlated than the "youngest" 10 per cent. Harker et al. (2006) studied the environmental dependence of halo formation times. They concluded that halos of a given mass in denser regions formed at higher redshifts than those in less dense regions. Maulbetsch et al. (2007) estimated that halos of $\sim 10^{12} M_{\odot}$ assemble $\sim 1.5$ Gyr earlier in high density regions than in low density regions. Using the Millennium simulation and arguments linking the formation of dark matter halos to that of both SMBHs and early quasars, Springel et al. (2005) also showed that the first quasars end up today as the central galaxies in rich clusters. As a result, nearby quasars in low density environments should be younger than quasars in high density environments.

Observationally, the clustering of high redshift quasars in the Sloan Digital Sky Survey was studied by Shen et al. (2007). Their results indicate that clustering increases for high redshift quasars with redshift. Using the 2dFQSO Redshift Survey (2QZ), Croom et al. (2005) showed that quasar clustering increases with redshift. This suggests that earlier quasars were located in higher density environments than the recent ones, and that high-redshift quasars can be used as beacons to search for high-density regions (Coldwell \& Lambas 2006). Our results for the location of nearby quasars agree with these observations.

Another interesting question is what is the relation between dark matter halos and their environment for the observed redshift distribution of the number of quasars per unit volume. This distribution for the quasar main-population reaches a maximum around $z \simeq 2-3$ both in X-ray selected and optically selected surveys (Silverman et al. 2005).

Maulbetsch et al. (2007) addressed the problem of how to explain the observed dependencies of galaxies on their environment (the morphology-density relation) with the mass assembly history of dark matter halos. They showed that the aggregation of mass onto the dark matter halos via halo mergers and mass accretion has a strong dependence on the environment and redshift. While halos in low density environments grow mainly by mass accumulation, in high density environments halo mergers are the dominant means of mass increase. The latter one is mainly determined by the properties of the subhalo population and the survival of subhalos in the main dark matter halo. The mass fraction accumulated by mergers is a function of redshift that decreases towards $z=0$. This is true for both low and high density environments, but the decrease is steeper in low density regions. At the same time, at $z=0$ mergers of low mass halos are relatively more numerous in low density environments. This is interesting because we know that quasar activity is usually expected to be triggered by galaxy interactions.

Finally, for supercluster scales, numerical simulations by Einasto et al. (2005) showed that in voids the dynamical evolution of halos is very slow and finally stops, while in supercluster regions the dynamical evolution continues until the present day. In superclusters, rich clusters at the core regions form first via halo mergers. In less dense regions of the superclusters and rich filaments, the halo evolution is suppressed compared to the core regions.

Thus, it is not a far-fetched idea that dark matter halos, and the evolution in the mass aggregation rate and its dependence on environment on all scales, play an important role in quasar evolution and their observed redshift distribution. The triggering mechanisms, which influence mass aggregation onto black holes, may have been suppressed because of environmental reasons, which are also related to the evolution of superclusters. Of course, local astrophysical processes related to baryonic matter must explain the observed properties of quasars in detail.

Taking into account halo-formation times and merger rates as a function of the halo mass and halo environment, also on supercluster scales, together with the estimated quasar duty-cycle, it should be possible to generate quasars in cosmological simulations, and to compare the results with the observed quasar distribution. We are planning to do this in a forthcoming paper.

\section{Conclusions}

We have studied the environments of quasars in the SDSS on different scales. Our main results can be summarized as follows:

(i) At the distances from 2 to $20 h^{-1} \mathrm{Mpc}$ from quasars the number density of galaxies is lower than the density at the same distances from galaxies. This effect is particularly strong for the brightest galaxies.

(ii) The groups of galaxies that have a quasar closer than $2 h^{-1} \mathrm{Mpc}$ are poorer and less luminous than all the groups in average. Also, groups that consist of four or more galaxies have lower richnesses and luminosities if there is a quasar closer than $10 \mathrm{~h}^{-1} \mathrm{Mpc}$.

(iii) The groups of galaxies that have a quasar neighbour at a distance of between 10 and $15 \mathrm{Mpc}$ are richer and more luminous than groups on average. The same result can also be seen if we examine the rich groups only.

(iv) Quasars avoid the densest regions in the (luminosity) density field, which correspond to rich supercluster cores. Nearby quasars are located on the outskirts of superclusters or in the filaments connecting them. Quasar evolution may be related to these highest density enhancements in the Universe.

Acknowledgements. We thank the anonymous referee for constructive comments that helped us improve the paper. This project was supported by the Finnish Academy funding. H. Lietzen was supported by Magnus Ehrnrooth foundation. E. Tago, E. Saar, J. Liivamägi, E. Tempel, M. Einasto, J. Einasto and M. Gramann were supported by the Estonian Science Foundation grant Nos. 6104 and 7146, and by the Estonian Ministry for Education and Science research project TO 0060058S98. Funding for the SDSS and SDSS-II has been provided by the Alfred P. Sloan Foundation, the Participating Institutions, the National Science Foundation, the U.S. Department of Energy, the National Aeronautics 
and Space Administration, the Japanese Monbukagakusho, the Max Planck Society, and the Higher Education Funding Council for England. The SDSS Web Site is http://www.sdss.org/. The SDSS is managed by the Astrophysical Research Consortium for the Participating Institutions. The Participating Institutions are the American Museum of Natural History, Astrophysical Institute Potsdam, University of Basel, University of Cambridge, Case Western Reserve University, University of Chicago, Drexel University, Fermilab, the Institute for Advanced Study, the Japan Participation Group, Johns Hopkins University, the Joint Institute for Nuclear Astrophysics, the Kavli Institute for Particle Astrophysics and Cosmology, the Korean Scientist Group, the Chinese Academy of Sciences (LAMOST), Los Alamos National Laboratory, the Max-PlanckInstitute for Astronomy (MPIA), the Max-Planck-Institute for Astrophysics (MPA), New Mexico State University, Ohio State University, University of Pittsburgh, University of Portsmouth, Princeton University, the United States Naval Observatory, and the University of Washington.

\section{References}

Adelman-McCarthy, J. K., Agüeros, M. A., Allam, S. S., et al. 2007, ApJS, 172, 634

Antonucci, R. 1993, ARA\&A, 31, 473

Bahcall, J. N., Schmidt, M., \& Gunn, J. E. 1969, ApJ, 157, 77

Balogh, M., Eke, V., Miller, C., et al. 2004, MNRAS, 348, 1355

Blanton, M. R., Hogg, D. W., Bahcall, N. A., et al. 2003, ApJ, 592, 819

Ceccarelli, L., Padilla, N. D., Valotto, C., \& Lambas, D. G. 2006, MNRAS, 373, 1440

Coldwell, G. V., \& Lambas, D. G. 2006, MNRAS, 371, 786

Constantin A., Hoyle F., \& Vogeley M. 2008, ApJ, 673, 715

Croom S., Boyle, B., Shanks, T., et al. 2005, MNRAS, 356, 415

De Robertis, M. M., Yee, H. K. C., \& Hayhoe, K. 1998, ApJ, 496, 93

Dressler, A. 1980, ApJ, 236, 351

Einasto, J., Hütsi, G., Einasto, M., et al. 2003, A\&A, 405, 425

Einasto, J., Tago, E., Einasto, M., et al. 2005, A\&A, 439, 45
Einasto, J., Einasto, M., Tago, E., et al. 2007a, A\&A, 462, 811 Einasto, M., Einasto, J., Tago, E., et al. 2007b, A\&A, 464, 815 Einasto, M., Saar, E., Martínez, V. J., et al. 2008, ApJ, 685, 83 Ferrarese, L. 2002, ApJ, 578, 90

Gao, L., Springel, V., \& White, S. D. M. 2005, MNRAS, 363, L66

Graham, A. W., Erwin, P., Caon, N., \& Trujillo, I. 2001, ApJ, 563, L11

Gottlöber, S., Lokas, E. L., Klypin, A., \& Hoffman, Y. 2003, MNRAS, 344, 715 Harker, G., Cole, S., Helly, J., Frenk, C., \& Jenkins, A. 2006, MNRAS, 367, 1039

Hopkins, P. F., Hernquist, L., Martini, P., et al. 2005a, ApJ, 625, L71

Hopkins, P. F., Hernquist, L., Cox. T. J., et al. 2005b, ApJ, 630, 705

Kauffmann, G., White, S. D. M., Heckman, T. M., et al. 2004, MNRAS, 353, 713

Li, C., Kauffmann, G., Wang, L., et al. 2006, MNRAS, 373, 457

Longair, M. S., \& Seldner, M. 1979, MNRAS, 189, 433

Magorrian, J., Tremaine, S., Richstone, D., et al. 1998, AJ, 115, 2285

Maulbetsch, C., Avila-Reese, V., Colin, P., et al. 2007, ApJ, 654, 53

McLure, R. J., \& Dunlop, J. S. 2001, MNRAS, 321, 515

McLure, R. J., \& Dunlop, J.S., 2004, MNRAS, 352, 1390

Miller, C. J., Nichol, R. C., Gómez, P. L., Hopkins, A. M., \& Bernardi, M. 2003, ApJ, 597, 142

Schneider, D. P., Hall, P. B., Richards, G. T., et al. 2007, AJ, 134, 102

Sellwood, J. A., \& Moore, E. M. 1999, ApJ, 510, 125

Shen, T., Strauss, M., Oguri, M., et al. 2007, AJ, 133, 2222

Silverman, J., Green P., Barkerhouse, R., et al. 2005, ApJ, 624, 630

Smith, E. P., O’Dea, C. P., \& Baum, S. A. 1995, ApJ, 441, 113

Springel V., White, S. D. M., Jenkins, A., et al. 2005, Nature, 435, 629

Strand, N. E., Brunner, R. J., \& Myers, A. D. 2008, ApJ, 688, 180

Tago, E., Einasto, J., Saar, E., et al. 2008, A\&A, 479, 927

Tempel, E., Einasto, J., Einasto, M., Saar, E., \& Tago, E. 2008, A\&A, 495, 37

Tremaine, S., Gebhardt, K., Bender, R., et al. 2002, ApJ, 574, 740

Turner, E. L., \& Gott, J. R., III 1976, ApJS, 32, 409

von Benda-Beckmann A. M., \& Müller V. 2008, MNRAS, 384, 1189

Wold, M., Lacy, M., Lilje, P. B., \& Serjeant, S. 2000, MNRAS, 316, 267

Wurtz, R., Stocke, J. T., Ellingson, E., \& Yee, H. K. C. 1997, ApJ, 480, 547 